Article

\title{
Structural Irreversibility and Enhanced Brittleness under Fatigue in Zr-Based Amorphous Solids
}

\author{
Peng Tong ${ }^{1,2}$, Despina Louca ${ }^{1, *}$, Gongyao Wang ${ }^{3}$, Peter K. Liaw ${ }^{3}$, Yoshihiko Yokoyama ${ }^{4}$, \\ Anna Llobet ${ }^{5}$, Hiroshi Kawaji ${ }^{6}$, Yiming Qiu ${ }^{7}$ and Yunfeng Shi ${ }^{8}$ \\ 1 Department of Physics, University of Virginia, VA 22904, USA \\ 2 Key Laboratory of Materials Physics, Institute of Solid State Physics, Chinese Academy of \\ Sciences, Hefei 230031, China; E-Mail: tongpeng@issp.ac.cn \\ 3 Department of Materials Science and Engineering, The University of Tennessee, Knoxville, \\ TN 37831, USA; E-Mails: gwang@utk.edu (G.W.); pliaw@utk.edu (P.K.L.) \\ 4 Institute of Materials Research, Tohoku University, Sendai 980-8577, Japan; \\ E-Mail:yy@imr.tohoku.ac.jp \\ 5 Los Alamos National Laboratory, Lujan Neutron Scattering Center, Los Alamos, NM 87545, USA; \\ E-Mail: allobet@lanl.gov \\ 6 Materials and Structures Laboratory, Tokyo Institute of Technology, 4259-R3-8 Nagatsuta-cho, \\ Midori-ku, Yokohama 226-8503, Japan; E-Mail: kawaji@msl.titech.ac.jp \\ 7 NIST Center for Neutron Research, Gaithersburg, MD 20899, USA and Department of Materials \\ Science and Engineering, University of Maryland, College Park, MD 20742, USA; \\ E-Mail: yiming.qiu@nist.gov \\ 8 Department of Materials Science and Engineering, Rensselaer Polytechnic Institute, Troy, \\ NY 12180, USA; E-Mail: shiy2@rpi.edu
}

* Author to whom correspondence should be addressed; E-Mail: louca@virginia.edu; Tel.: +1-434-924-6802; Fax: +1-434-924-4576.

Received: 16 August 2012; in revised form: 9 November 2012 / Accepted: 11 December 2012 / Published: 19 December 2012

\begin{abstract}
The effect of fatigue on $\mathrm{ZrCuAl}$ amorphous metals induced by mechanical cyclic loading is investigated using inelastic neutron scattering and the pair density function analysis of neutron diffraction data. With cooling, the local atomic structure undergoes reorganization under fatigue that is directly related to the number of fatigue cycles. Also under fatigue, suppression in the atomic dynamics is observed as well. A structural restructuring occurs within a $4 \AA$ radius and intensifies with increasing the compression cycles, whereas the vibrational density of states is attenuated as the intensity
\end{abstract}


shifts towards the elastic, zero-energy transfer peak. The combined static and dynamic structural effects are a signature of the microscopic changes brought about by fatigue, and together may be the onset for subsequent behaviors following extended cyclic loading such as fracture. Even after the load is removed, the structural changes described here remain and increase with repeated cyclic loading which is an indication that the lattice deforms even before shear bands are formed.

Keywords: local structure; compression fatigue; dynamics; VDOS attenuation

\section{Introduction}

Amorphous metals are solids devoid of periodicity, prepared by the very fast cooling of a viscous liquid to avoid crystallization. Trapped in this supercooled state with an atomic topology that resembles that of the liquid [1], amorphous alloys exhibit uniquely different metallurgy from crystals, and have widespread industrial implications. With strengths that can surpass those found in crystalline counterparts, how they mechanically respond and deform under applied stresses is central to their functionality. Typically, metallic glasses have undesirably low ductility, though, and most commonly plastically deform through shear bands [2-5]. The structure, which is usually perceived as short-ranged in nature, and how it deforms under stress is clearly important in controlling plastic flow [6]. However its role and that of its dynamics are still poorly understood.

In spite of their high strengths [7] glassy metals are often brittle [8] like ceramics, instead of crystalline metals that are ductile, and permanently deform by forming shear bands under stresses. In the present work, we investigate the relation between the vibrational properties in the glassy state and the atomic properties leading to plastic flows [9] under mechanical testing such as compression fatigue. The system used for the purpose of addressing this question is a strong glass former, $\mathrm{ZrCuAl}$ [10-12]. The impact from fatigue on the nature of static disorder and low temperature dynamics are investigated via neutron scattering.

Amorphous matter exhibits unusual low temperature characteristics [13] that are distinctly different from any crystalline equivalent. One anomaly is the observation of excess vibrational density of states manifested in the so-called boson peak, and observed by techniques such as neutron [14] and Raman scattering [15]. This is also manifested in an unusual low temperature behavior of the heat capacity, $C_{P}$, as well, in which $C_{P}$ deviates from the $\mathrm{T}^{3}$ Debye law expected of a typical metallic solid with cooling. The boson peak is a universal attribute in glassy systems. Its origin has been linked to transverse vibrational modes associated with defective structures [16], to local vibrational modes of clusters [17], and to crossover dynamics of local and acoustic modes [18]. More recently it was proposed that the boson peak is analogous to the acoustic van Hove singularity in crystals [19-21]. The excess vibrational density of states, as determined both from the neutron inelastic scattering and $C_{P}$ measurements displays a maximum of a few milli-electron Volts $(\mathrm{meV})$ (of the order of $\left.10^{-12} \mathrm{~s}\right)$. The intensity of the boson peak has in turn been directly related to other concepts such as fragility [1,22] and to the local free volume [16], which has been well characterized in molecular glasses [23]. However, little is known regarding the nature of the boson peak in metallic glasses with isotropic 
structures and even less so under the influence of mechanical deformation. With only a few of the alloys discovered thus far exhibiting good mechanical properties [24,25], it would be important to investigate the relation of static disorder and atomic dynamics to the mechanical deformation brought upon by fatigue.

Fatigue is critical in applications such as springs, in some sensors, actuators, and microelectrochemical systems that require lifetimes of $10^{12}$ cycles if not more [26]. However, fatigue damage can occur in metallic glasses at very low stresses, sometimes as low as $10 \%$ of the yield stress [27], limiting their use in structural applications [26,27]. Understanding the relation of the atomic structure to the mechanical deformation behavior brought upon by a static load is a challenging issue. That is because bulk metallic glasses can deform both homogeneously and inhomogeneously. At low temperatures, the deformation is inhomogeneous where plastic flow concentrates on shear bands, accompanied by a decrease of the viscosity and an increase of the free volume leading to strain softening [28]. Shear bands are observed under fatigue conditions due to fatigue softening [29]. It has been suggested that the formation of shear bands and fatigue softening are linked to the fracture of bulk metallic glasses during the fatigue process [30].

In this paper, we show that under high-cycle fatigue, the ternary $\mathrm{ZrCuAl}$ glass changes in part due to an atomic restructuring that suppresses fluctuations. A local reorganization of the atom packing is observed, where the changes intensify as the number of compression cycles increases. This is best observed by cooling the system down to low enough temperatures that reduces the smearing induced by thermal vibrations. Associated with these changes in the static structure is a softening of the lattice dynamics. The attenuation implies an increase in the sound velocity, suggesting that the glass becomes stiffer and more likely brittle under fatigue.

\section{Results and Discussion}

\subsection{Specific Heat}

The $\mathrm{Zr}$ based ternary glass is a model system with exceptional glass forming ability. The low-temperature $C_{P}$ anomaly associated with the presence of an excess vibrational low frequency contribution is shown in Figure 1a for $\mathrm{Zr}_{60} \mathrm{Cu}_{30} \mathrm{Al}_{10}$ and $\mathrm{Zr}_{50} \mathrm{Cu}_{40} \mathrm{Al}_{10}$. Plotted in the figure is $C_{P}$ with the electronic contribution, $\gamma T$, subtracted and then normalized by $T^{3}$ [27]. Such normalization should have rendered a temperature independent curve for a solid that follows the Debye model of $C_{P}$, but instead a bump is observed that reaches a peak around $10 \mathrm{~K}$. This behavior has been previously observed in metallic and other types of glassy systems and is associated with strong glasses [27,31]. While the bump is present in both compositions, it is distinctly more intense in $\mathrm{Zr}_{60} \mathrm{Cu}_{30} \mathrm{Al}_{10}$ that in turn may indicate that the amplitude of excess vibrational density of states is higher in this alloy. To reproduce the bump, the $C_{P}$ is typically fit by two terms, one originating from the Debye model of the $C_{P}$ and an Einstein term of a single oscillator with an Einstein temperature, $\theta_{\mathrm{E}} \sim 56 \mathrm{~K}$ for $\mathrm{Zr}_{60} \mathrm{Cu}_{30} \mathrm{Al}_{10}$ $\left(\theta_{\mathrm{E}} \sim 63 \mathrm{~K}\right.$ for $\left.\mathrm{Zr}_{50} \mathrm{Cu}_{40} \mathrm{Al}_{10}\right)$. By combining both terms, the resulting curve reasonably reproduces the bump at low temperatures as seen in Figure 1a. An Einstein oscillator represents a localized vibrational mode, and although rare in closed-packed structures, is ubiquitous in compounds with large voids or cage structures [32,33]. It has been suggested that atoms occupying these voids in metallic glasses are 
loosely bound and are thus free to rattle almost independently, giving rise to single oscillator modes. Also shown in Figure $1 \mathrm{~b}$ is the $C_{P}$ for the same sample after fatigue. Very small differences can be discerned between the dashed (post-fatigue) and solid (pre-fatigue) lines. The $C_{P}$ temperature dependence of the $\mathrm{Zr}_{50} \mathrm{Cu}_{40} \mathrm{Al}_{10}$ alloy shows a very small enhancement but no visible differences can be seen in $\mathrm{Zr}_{60} \mathrm{Cu}_{30} \mathrm{Al}_{10}$.

Figure 1. (a) The heat capacity at constant pressure, $C_{P}$, with $\gamma \mathrm{T}$ subtracted and normalized by $T^{3}$ is plotted as a function of temperature for the two compositions. $\gamma$ is the Sommerfeld constant; (b) The Debye, $C_{\mathrm{D}}$ and Einstein, $C_{\mathrm{E}}$, terms are estimated. They are compared to the experimental $C_{P}$.

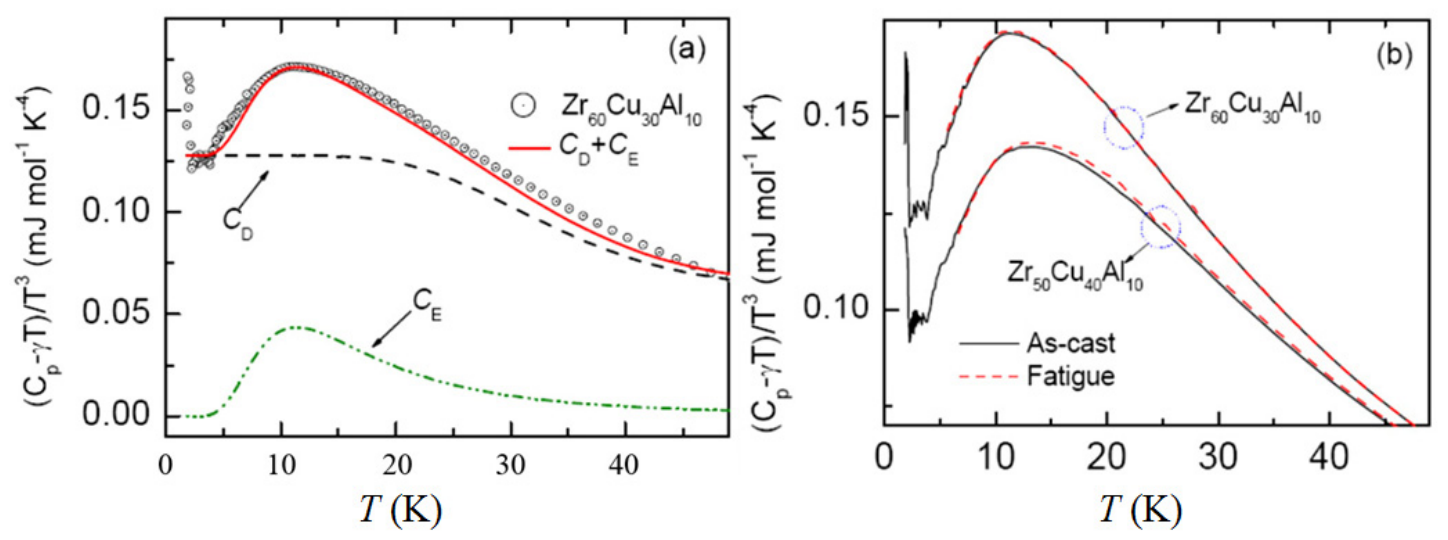

\subsection{Inelastic Neutron Scattering}

Shown in Figure 2a is the $S(Q)$ from data collected at $300 \mathrm{~K}$ for $\mathrm{Zr}_{60} \mathrm{Cu}_{30} \mathrm{Al}_{10}$ and obtained from a diffraction experiment (solid line). The broad peaks are a signature of the static disorder present in the glass. This $S(Q)$ is used in the PDF analysis that will be discussed further below. Also shown in the same figure is the structure function obtained from the inelastic experiment but with the data integrated over the elastic peak from $-2 \mathrm{meV}$ to $2 \mathrm{meV}$ (red symbols). The structure function obtained from the diffraction experiment extends to very high momentum transfer, $Q$, while the one obtained from the inelastic experiment is limited in $Q$, but the main features are reproduced as in the diffraction measurement.

Shown in Figure $2 \mathrm{~b}$ is the energy, $E-Q$ map collected at $T=300 \mathrm{~K}$ for $\mathrm{Zr}_{60} \mathrm{Cu}_{30} \mathrm{Al}_{10}$ at the DCS. The red bar corresponds to the elastic scattering that has the brightest intensity. The intensity integrated in energy from -2 to $2 \mathrm{meV}$, the breadth of the elastic peak, yields the elastic $S(Q)$ shown in Figure 2a. Beyond the elastic peak, an inelastic continuum is observed below $2 \AA^{-1}$ in addition to two strong localized modes. The experimental setup is such that most of the information we obtain will be in the neutron energy gain side $(-\mathrm{meV})$ and this is what is shown in the figure. The neutron energy loss side $(+\mathrm{meV})$ extends up about $10 \mathrm{meV}$. However, the elastic line is asymmetric, with the asymmetry appearing on the right side of the peak and most like due to the sample alignment. Because of this, the analysis was focused on the neutron gain side. Of the two modes, the rather narrow one is centered at $Q \sim 2.5 \AA^{-1}$ and $E_{1} \sim 5 \mathrm{meV}$ and appears at the same median $Q$-position as the strongest peak in the static $S(Q)$. Thus clearly the correlations associated with the tallest peak in the $S(Q)$ have the corresponding fluctuations in energy. Similarly, the broader excitation extends beyond $Q \sim 4 \AA^{-1}$ with an $E_{2} \sim 15 \mathrm{meV}$ 
and appears in the same $Q$-region as for the second broad peak in the $S(Q)$ of Figure 2a. By integrating the data over all accessible momentum transfers, the dynamic structure function, $S(\hbar \omega)$, shown in Figure 2c is obtained. The $S(\hbar \omega)$ is normalized by the Bose factor appropriate for the neutron energy gain side, $[n(\omega)+1]$. The integration overall all $Q$ incorporates all excitations seen in Figure $2 \mathrm{~b}$ and shown in this figure is their temperature dependence. It has been previously suggested that such collective excitations result from acoustic, phonon-like modes arising from a pseudo-Brillouin zone due to some dynamic periodicity [34,35]. Below $50 \mathrm{~K}$, the peaks are not discernible because of the low population occupancy, while their intensity increases following Bose-Einstein statistics. At $50 \mathrm{~K}$, the $E_{1}$ peak at $\sim 5 \mathrm{~K}$ is quite pronounced while $E_{2} \sim 15 \mathrm{meV}$ is suppressed. Above $50 \mathrm{~K}, E_{1}$ gains in intensity and by $300 \mathrm{~K}$, it appears to be almost as intense as $E_{1}$. Shown in Figure $2 \mathrm{~d}$ is the integration of the data in three different $Q$-slices corresponding to the three different regions in the $E-Q$ contour map: from 0 to $2 \AA^{-1}$ (low- $Q$ ), from 2 to $4 \AA^{-1}$ (intermediate- $Q$ ) and from 4 to $7 \AA^{-1}$ (high- $Q$ ) for the two compositions studied. In all the curves, the intensity for $\mathrm{Zr}_{50} \mathrm{Cu}_{40} \mathrm{Al}_{10}$ is slightly lower than that for $\mathrm{Zr}_{60} \mathrm{Cu}_{30} \mathrm{Al}_{10}$. Typically the phonon energy spectrum is normalized by the $\sqrt{\mathrm{M}}$ (the total mass) and hence as expected, the spectrum for the $\mathrm{Zr}_{60} \mathrm{Cu}_{30} \mathrm{Al}_{10}$ is shifted closer to the elastic line. With temperature, $E_{1}$ remains essentially constant for both compositions, while $E_{2}$ shifts to lower energies by cooling from $150 \mathrm{~K}$, indicating some kind of softening associated with these fluctuations.

Figure 2. (a) The static $S(Q)$ determined from diffraction compares well to the elastic $S(Q)$ determined from the inelastic measurement. The small peaks observed in the elastic $S(Q)$ are from Al; (b) The energy versus momentum transfer map shows the presence of the two excitations. A broad continuum is also observed at very low $Q ;(\mathbf{c})$ The total $S(\hbar \omega)$ as a function of temperature for $\mathrm{Zr}_{60} \mathrm{Cu}_{30} \mathrm{Al}_{10}$; (d)The $S(Q, \omega)$ determined at three regions of momentum space, low- $Q$, intermediate- $Q$ and high- $Q$ and compared between the two compositions studied.
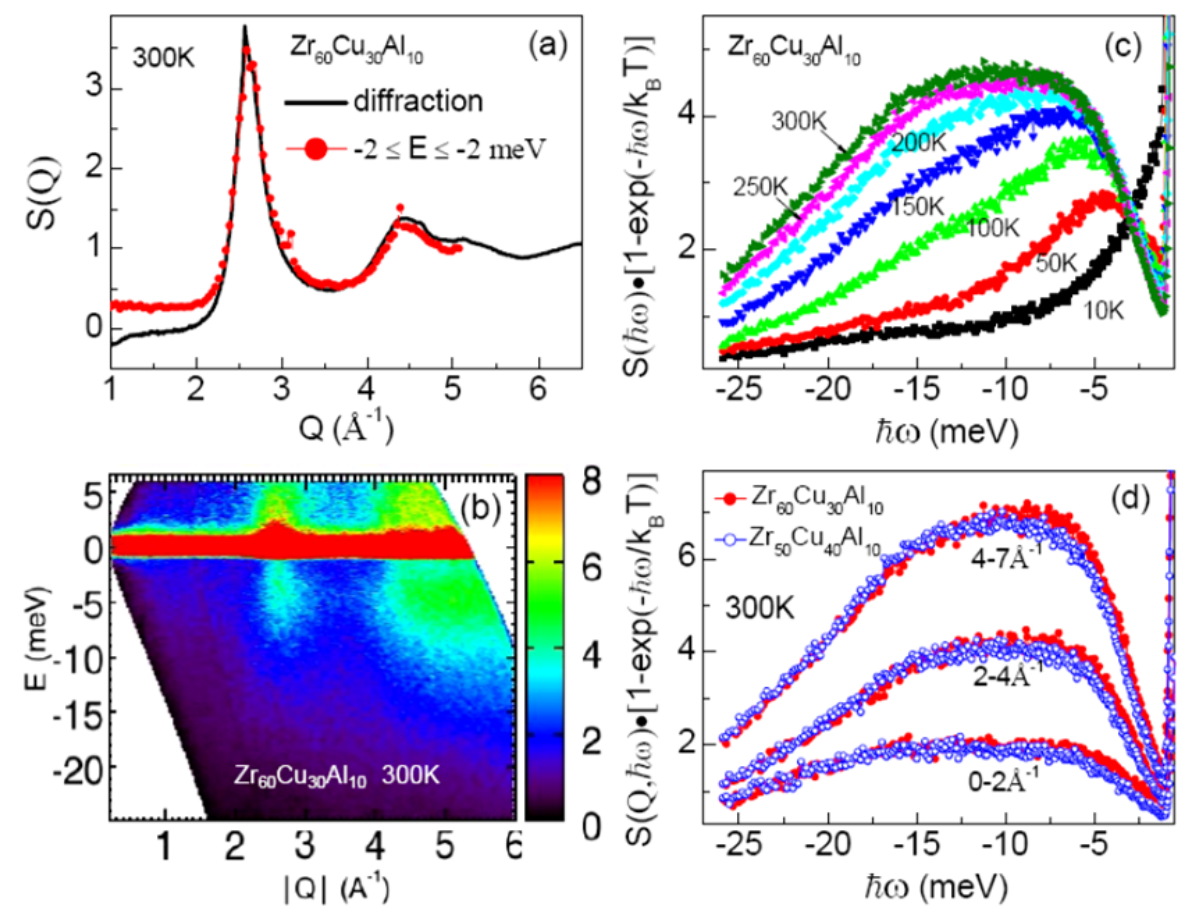


\subsection{Vibrational Density of States under Fatigue}

What happens to the vibrational density of states (VDOS) intensity under fatigue? Shown in Figure $3 \mathrm{a}$ is the VDOS/ $E^{2}$ for the two $\mathrm{Zr}_{50} \mathrm{Cu}_{40} \mathrm{Al}_{10}$ alloys, one as-cast and the after fatigue. The VDOS function is given by $Z(\omega)=\left((S(Q, \omega) \omega) /\left(Q^{2}[n(\omega)+1]\right)\right)$. An applied maximum stress of $1600 \mathrm{MPa}$ was used at $10^{5}$ compression cycles. Data were collected at two temperatures, 300 and $150 \mathrm{~K}$. The amount of sample used for each experiment was different ( $50 \mathrm{~g}$ of the as-cast sample versus $10 \mathrm{~g}$ of the fatigue sample). Hence the data for each set of samples were normalized by the integrated intensity of the elastic peak and the high energy spectrum end was used to normalize the background. At constant temperature, the inelastic intensity is suppressed after fatigue while the intensity shifts towards the elastic line. The as-cast alloy consistently shows a higher intensity at both temperatures than the alloy after mechanical testing. Fatigue decreases the number of vibrational modes contributing to these excitations, in an analogous way to freezing of these modes as they become static. Shown in Figure $3 b$ is the $\mathrm{VDOS} / E^{2}$ at $300 \mathrm{~K}$ only and selectively integrated in the three $Q$ regions defined earlier. In the low- $Q$ and intermediate- $Q$ regions, the intensity is clearly reduced after fatigue, and shifts to the elastic peak. However, in the high- $Q$ region from 2 to $4 \AA^{-1}$, the intensity appears to change little with fatigue. This trend may indicate that the fluctuations mostly affected by fatigue involve long wavelength cluster correlations pertaining to shear bands.

Figure 3. (a) The $\mathrm{VDOS} / E^{2}$ for $\mathrm{Zr}_{50} \mathrm{Cu}_{40} \mathrm{Al}_{10}$ is shown for the data collected for an as-cast alloy and one that has undergone $10^{5}$ compression cycles. The inelastic intensity is suppressed at both temperatures shown. (b) The $S(Q, \omega)$ is determined at three regions of momentum space, low- $Q$, intermediate- $Q$ and high- $Q$ and the comparison is for data collected before and after fatigue.
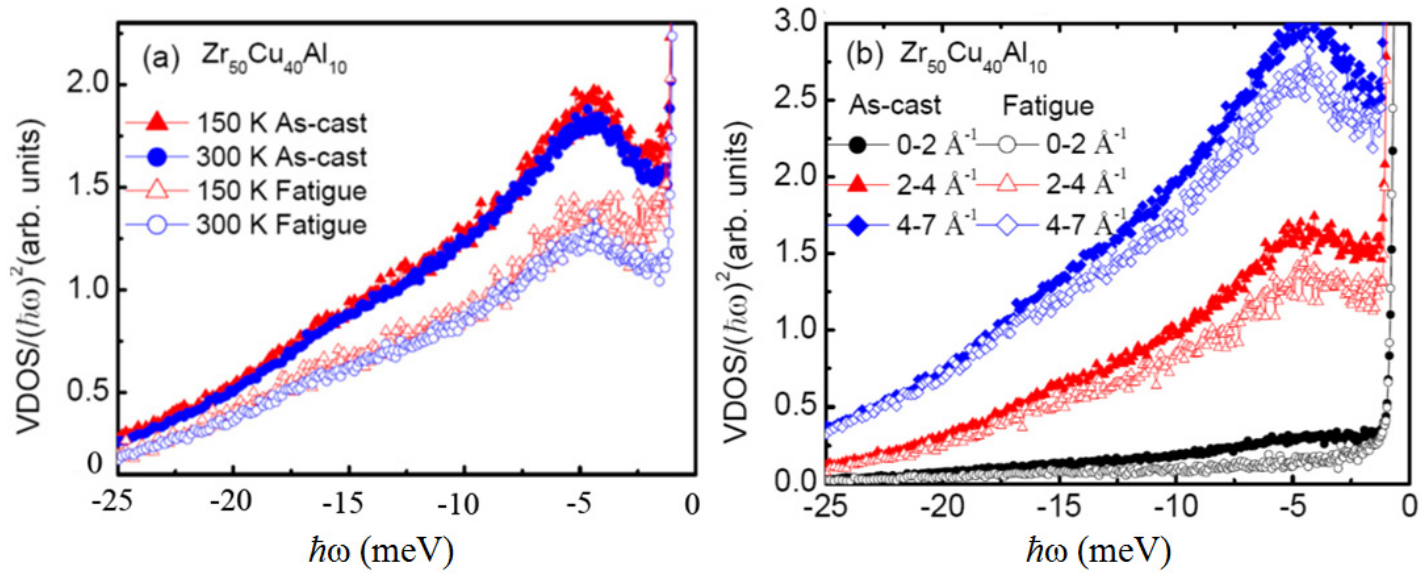

\subsection{Local Structure under Fatigue}

The suppression of these excitations, similarly to the suppression of the boson peak [36] by external stimuli, may be linked to the structural re-organization that we observed in the local structure. Following a well established procedure of Fourier transforming the static $S(Q)$ from the reciprocal space into a real space representation, the pair density function (PDF) [37] is obtained and the results are shown in Figure 4. The PDF involves the integration over a finite energy window $(\sim 10 \mathrm{meV})$ and 
represents a time-averaged local structure, in the absence of an underlying periodicity. The neutron diffraction experiments were performed from 300 down to $10 \mathrm{~K}$ on as-cast alloys and under varying compression-compression fatigue cycles on the two compositions. The PDF up to $6 \AA$ as a function of fatigue cycles is shown in Figure $4 \mathrm{a}$ for $\mathrm{Zr}_{60} \mathrm{Cu}_{30} \mathrm{Al}_{10}$ at a constant temperature of $10 \mathrm{~K}$. With increasing the number of cycles, the height of the first peak rises while its width becomes narrow and its shape changes. A similar observation is made by cooling the structure from 300 to $10 \mathrm{~K}$ of the alloy subjected to $10^{5}$ cycles (Figure $4 \mathrm{~b}$ ). Given that the area under the PDF peaks is conserved, it is not unusual for the peaks to become narrower and taller with cooling because cooling reduces the atomic thermal vibrations that contribute to the peak broadening. However, it is unusual for the peaks to change shape, as seen in both Figure 4a,b. It is even more unusual for the intensity to change at a constant temperature as seen in the PDF's of Figure 4a as a function of fatigue. Also shown in this figure are three partial functions, corresponding to the $\mathrm{Zr}-\mathrm{Zr}, \mathrm{Zr}-\mathrm{Cu}$ and $\mathrm{Cu}-\mathrm{Cu}$ atom correlations that contribute the most to the total PDF, and calculated from an embedded atom potential model $[38,39]$. The increase in the peak sharpness corresponds to the structure becoming more compact as the peak width narrows, and signals the reduction of free volume that may in turn be linked to brittleness.

Figure 4. (a) The PDF corresponding to the local atomic structure of $\mathrm{Zr}_{60} \mathrm{Cu}_{30} \mathrm{Al}_{10}$ under different fatigue cycles at $10 \mathrm{~K}$. Also shown are partial PDF's for select pairs obtained from molecular dynamics. (b) The local atomic structure as a function of temperature for the alloy at $10^{5}$ cycles. (c) The HWHM of the first PDF peak as a function of temperature for 4 different fatigue cycles. The inset shows the HWHM at two temperatures. (d) The change of the first PDF peak height with temperature at four fatigue cycles. The inset shows the change of the peak height at two temperatures.
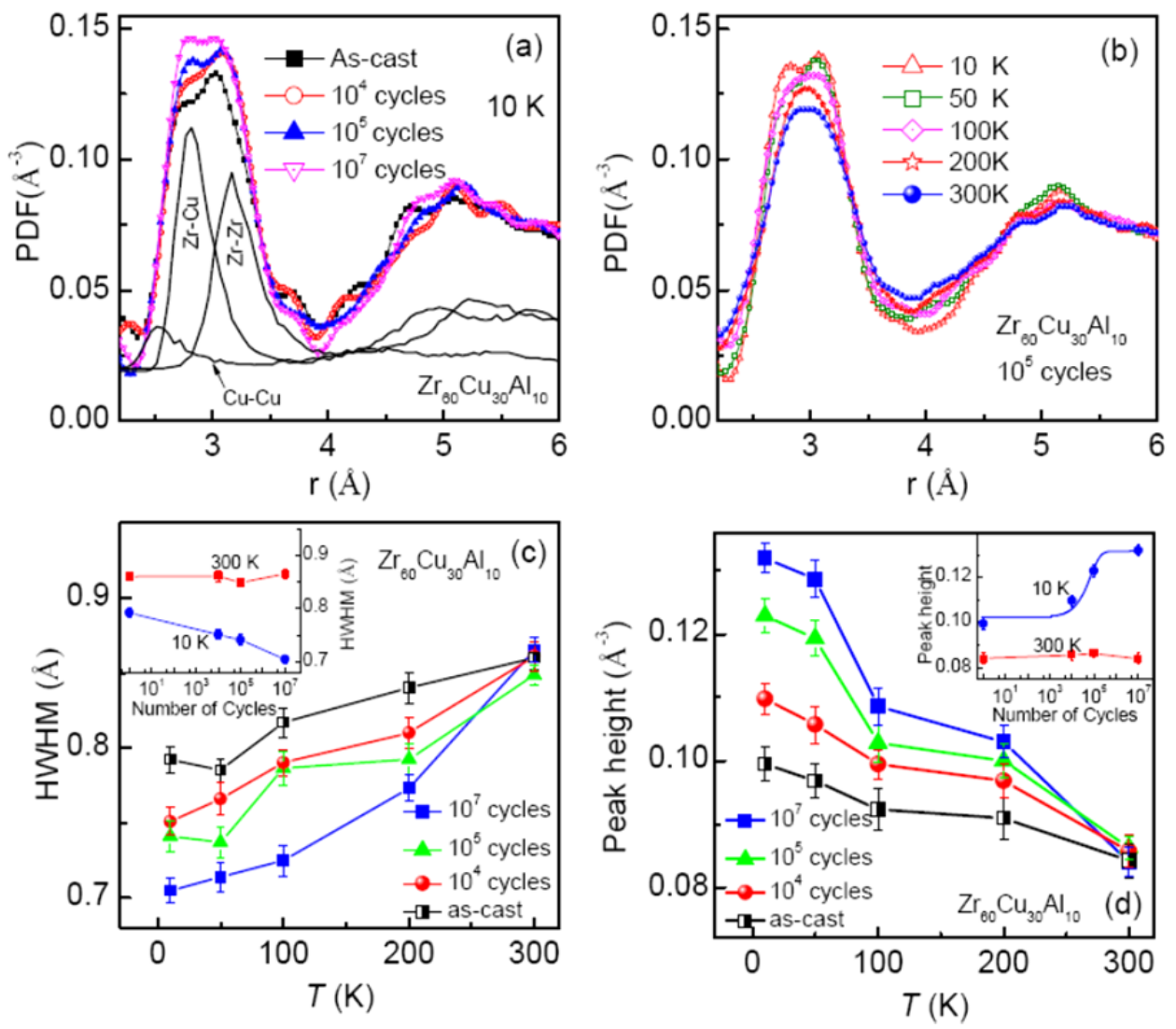
The reduction of the half-width-half-max (HWHM) of the first peak, shown in Figure 4c, indicates that the first coordination sphere contracts upon fatigue loading. The changes are most noticeable at low temperatures than at room temperature (see inset of this figure) because of the reduction of thermal vibration, while a clear correlation is observed between the reduction of HWHM and fatigue cycles. A hysteresis is observed on warming where the system returns to its original state around $100 \mathrm{~K}$ (not shown). If we consider the temperature dependence of the as-cast sample as the baseline (black symbols), it is clear that the effects from fatigue are above this baseline. The change in the peak height of the first PDF peak at this maximum $1600 \mathrm{MPa}$ stress level is most dramatic as the load cycles increase as shown in Figure 4d. In the inset of this figure, the data points at $10 \mathrm{~K}$ can be fit by an exponential function although there are only 4 points. But in comparison to the constant behavior observed at $300 \mathrm{~K}$, the rapid peak height change at $10 \mathrm{~K}$ is clear. Thus permanent changes are observed in the local atomic structure in response to the presence of residual stresses even after the load is removed. On the other hand, at $300 \mathrm{~K}$, the response remains almost constant where the effects are masked by the thermal vibration of atoms.

\section{Experimental Section}

The alloys of $\mathrm{Zr}_{50} \mathrm{Cu}_{40} \mathrm{Al}_{10}$ and $\mathrm{Zr}_{60} \mathrm{Cu}_{30} \mathrm{Al}_{10}$ (in atomic percent) were prepared using a ladle-hearth type arc-melt tilt-casting technique. The ingots were cut into $6.0 \mathrm{~mm} \times 6.0 \mathrm{~mm}$ cylindrical specimens for compression-compression fatigue measurements. The two end sides of each alloy were polished using a $1200 \mathrm{SiC}$ grit-surface finish perpendicular to the longitudinal axis of the specimens using a polishing fixture to ensure that the sides were parallel to one another. A computer-controlled Material Test System (MTS) servohydraulic-testing machine was employed for fatigue studies. The sample was tested with a load ratio, $R$, of 10 where $R=\sigma_{\min } / \sigma_{\max }$ (where $\sigma_{\min }$ and $\sigma_{\max }$ are the applied minimum and maximum stresses, respectively) under a load-control mode, using a sinusoidal waveform at a frequency of $10 \mathrm{~Hz}$. The neutron powder diffraction measurements were carried out at the Los Alamos National Laboratory using the high intensity powder diffractometer (HIPD). Data were collected in a closed-cycle refrigeration system as a function of temperature. The structure function, $S(Q)$, obtained from the neutron powder diffraction data was Fourier transformed to obtain the pair density function (PDF) analysis. The inelastic data were collected using the Disk Chopper Spectrometer (DCS) at the NIST Center for Neutron Research (NCNR) as a function of temperature. Two incident energies were used, $\lambda_{\mathrm{i}}=2.2$ and $3.6 \AA$ while data before and after fatigue were collected for the $\mathrm{Zr}_{50} \mathrm{Cu}_{40} \mathrm{Al}_{10}$ composition only.

\section{Conclusions}

From the above results, the following conclusion can be drawn regarding the effects of mechanical fatigue on the atomic properties. A connection is present between the changes observed in the local structure and the dynamics in the solid state. The consequence of the increase of the first PDF peak height with cooling above the expected increase from thermal contraction is twofold: it signals a local restructuring due to microscopic changes as well as reduction of the VDOS. The local restructuring occurs because the lattice changes irreversibly, even after the load is removed. With repeated loading and unloading the glass eventually breaks, thus such lattice irreversibility is a precursory effect to 
failure. The decrease in the intensity of the vibrational spectrum and its shift towards the elastic channel follow the local restructuring. The local structure reorganization that we observe with modifications in the pair correlations amplitudes will have a direct effect on the normal modes and can explain the reduction of the amplitude of fluctuations with fatigue. It is the case that the system becomes brittle after fatigue and thus, brittleness is correlated to the suppression of the vibrational spectrum.

\section{Acknowledgments}

The work at the University of Virginia is supported by the National Science Foundation, under contract DMR-0804801, at the University of Tennessee under contracts DMR-0909037, CMMI-0900271 and 1100080, and at NIST, DCS is supported under contract DMR-0944772. The Los Alamos National Laboratory is operated by Los Alamos National Security LLC under DOE contract DE-AC52-06NA25396.

\section{Conflict of Interest}

The authors declare no conflict of interest.

\section{References}

1. Debenedetti, P.G.; Stillinger, F.H. Supercooled liquids and the glass transition. Nature 2001, 410, 259-267.

2. Spaepen, F. A microscopic mechanism for steady state inhomogeneous flow in metallic glasses. Acta Mater.1977, 25, 407-415.

3. Argon, A.S. Plastic deformation in metallic glasses. Acta Mater. 1979, 27, 47-58.

4. Masumoto, T.; Maddin, R. Structural stability and mechanical properties of amorphous metals. Mater. Sci. Eng. 1975, 19, 1-24.

5. Greer, A.L.; Ma, E. Bulk metallic glasses: At the cutting edge of metals research. MRS Bull. 2007, 32, 611-615.

6. Chen, M.; Inoue, A.; Zhang, W.; Sakurai, T. Extraordinary plasticity of ductile bulk metallic glasses. Phys. Rev. Lett. 2006, 96, 245502:1-245502:4.

7. Scopigno, T.; Ruocco, G.; Sette, F.; Monaco, G. Is the fragility of a liquid embedded in the properties of its glass? Science 2003, 302, 849-852.

8. Das, J.; Tang, M.B.; Kim, K.B.; Theissmann, R.; Baier, F.; Wang, W.H.; Eckert, J. "Work-hardenable" ductile bulk metallic glass. Phys. Rev. Lett. 2005, 94, 205501:1-205501:4.

9. Greer, A.L. Metallic glasses. Science 1995, 267, 1947-1953.

10. Freels, M.; Wang, G.Y.; Zhang, W.; Liaw, P.K.; Inoue, A. Cyclic compression behavior of a $\mathrm{Cu}-\mathrm{Zr}-\mathrm{Al}-\mathrm{Ag}$ bulk metallic glass. Intermetallics 2011, 19, 1174-1183.

11. Wang, G.Y.; Liaw, P.K.; Jin, X.Q.; Yokoyama, Y.; Huang, E.W.; Jiang, F.; Keer, L.M.; Inoue, A. Fatigue initiation and propagation behavior in bulk metallic glasses under a bending load. J. Appl. Phys. 2010, 108, 113512:1-113512:7. 
12. Wang, G.Y.; Qiao, D.C.; Yokoyama, Y.; Freels, M.; Inoue, A.; Liaw, P.K. Effects of loading modes on the fatigue behavior of Zr-based bulk metallic glasses. J. Alloys Comp. 2009, 483, 143-145.

13. Buchenau, U.; Nucker, N.; Dianoux, A.J. Neutron scattering study of the low-frequency vibrations in vitreous Silica. Phys. Rev. Lett. 1984, 53, 2316:1-2316:4.

14. Sette, F.; Krisch, M.H.; Masciovecchio, C.; Ruocco, G.; Monaco, G. Dynamics of glasses and glass-forming liquids studies by inelastic x-ray scattering. Science 1998, 280, 1550-1555.

15. Shintani, H.; Tanaka, H. Universal link between the boson peak and transverse phonons in glass. Nature Mat. 2008, 7, 870-877.

16. Sokolov, A.P.; Calemczuk, R.; Salce, B.; Kisliuk, A.; Quitmann, D.; Duval, E. Low temperature anomalies in strong and fragile glass formers. Phys. Rev. Lett. 1997, 78, 2405:1-2405:1.

17. Duval, E.; Boukenter, A.; Achibat, T. Vibrational dynamics and the structure of glasses. J. Phys. Condens. Matter. 1990, 2, 10227-10234.

18. Klinger, M.I.; Kosevich, A.M. Soft-mode-dynamics model of acoustic-like high-frequency excitations in boson-peak spectra of glasses. Phys. Lett. A 2001, 280, 365-370.

19. Chumakov, A.I.; Monaco, G.; Monaco, A.; Crichton, W.A.; Bosak, A.; Rüffer, R.; Meyer, A.; Kargl, F.; Comez, L.; Fioretto, D.; et al. Equivalence of the Boson peak in glasses to the transverse acoustic van hove singularity in crystals. Phys. Rev. Lett. 2011, 106, 225501:1-225501:4.

20. Schirmacher, W.; Diezemann, G.; Ganter, C. Harmonic vibrational excitations in disordered solids and the "Boson peak". Phys. Rev. Lett. 1998, 81, 136:1-136:4.

21. Taraskin, S.N.; Loh, Y.L.; Natarajan, G.; Elliott, S.R. Origin of the Boson peak in systems with lattice disorder. Phys. Rev. Lett. 2001, 86, 1255:1-1255:4.

22. Novikov, V.N.; Sokolov, A.P. Poisson's ratio and the fragility of glass-forming liquids. Nature 2004, 431, 961-963.

23. Johnson, W.L. Bulk Glass-Forming Metallic Alloys: Science and Technology. In Bulk Metallic Glasses, Proceedings of Materials Research Society Symposium, Boston, MA, USA, December 1-3, 1998; Johnson, W.L., Inoue, A., Liu, C.T., Eds.; Materials Research Society: Warrendale, PA, USA, 1999; Volume 554, pp. 191-196.

24. Inoue, A. Bulk Amorphous Alloys: Practical Characteristics and Applications; Trans Tech Publications: Zurich, Switzerland, 1999.

25. Pacard, C.E.; Homer, E.R.; Al-Aqeeli, N.; Schuh, C.A. Cyclic hardening of metallic glasses under Hertzian contacts: Experiments and STZ dynamics simulations. Philos. Mag. 2010, 90, 1373-1390.

26. Packard, C.E.; Witmer, L.M.; Schuh, C.A. Hardening of a metallic glass during cyclic loading in elastic range. Appl. Phys. Lett. 2008, 92, 171911:1-171911:3.

27. Li, Y.; Bai, H.Y.; Wang, W.H.; Samwer, K. Low-temperature specific heat anomalies associated with the boson peak in CuZr-based bulk metallic glasses. Phys. Rev. B 2006, 74, 052201:1-052201:4.

28. Inoue, A. Stabilization of metallic supercooled liquid and bulk amorphous alloys. Acta Mater. 2000, 48, 279-306.

29. Wang, G.Y.; Liaw, P.K.; Peter, W.H.; Yang, B.; Yokoyama, Y.; Benson, M.L.; Green, B.A.; Kirkham, M.J.; White, S.A.; Saleh, T.A.; et al. Fatigue behavior of bulk metallic glasses. Intermetallics 2004, 12, 885-892.

30. Qiao, D.C.; Huang, L.; Jiang, W.; Fan, C.; Liaw, P.K. Fatigue softening of $\left(\mathrm{Zr}_{58} \mathrm{Ni}_{13.6} \mathrm{Cu}_{18} \mathrm{Al}_{10.4}\right)_{99} \mathrm{Nb}_{1}$ bulk metallic glass. Metall. Mat. Trans. A 2010, 41, 1787-1791. 
31. Tang, M.B.; Bai, H.Y.; Pan, M.X.; Zhao, D.Q.; Wang, W.H. Einstein oscillator in highly-random-packed bulk metallic glass. Appl. Phys. Lett. 2005, 86, 021910:1-021910:3.

32. Keppens, V.; Mandrus, D.; Sales, B.C.; Chakoumakos, B.C.; Dai, P.; Coldea, R.; Maple, M.B.; Gajewski, D.A.; Freeman, E.J.; Bennington, S. Localized vibrational modes in metallic solids. Nature 1998, 395, 876-878.

33. Hermann, R.P. Einstein oscillators in thallium filled antimony skutterudites. Phys. Rev. Lett. 2003, 90, 135505:1-135505:1.

34. Grigera, T.S.; Martin-Mayor, V.; Parisi, G.; Verrocchio, P. Phonon interpretation of the "Boson peak" in supercooled liquids. Nature 2003, 422, 289-292.

35. Malinovsky, V.K.; Sokolov, A.P. The nature of boson peak in Raman spectra in glasses. Solid State Comm. 1986, 57, 757-761.

36. Yannopoulos, S.N.; Andrikopoulos, K.S.; Ruocco, G. On the analysis of the vibrational Boson peak and low-energy excitations in glasses. J. Non-Cryst. Solids 2006, 352, 4541-4551.

37. Kazimirov, V.Y.; Louca, D.; Widom, M.; Gu, X.-J.; Poon, S.J.; Shiflet, G.J. Local organization and atomic clustering in multicomponent amorphous steels. Phys. Rev. B 2008, 78, 054112:1-054112:5.

38. Daw, M.S.; Baskes, M.I. Semiempirical, quantum mechanical calculation of hydrogen embrittlement in metals. Phys. Rev. Lett. 1983, 50, 1285:1-1285:4.

39. Cheng, Y.Q.; Ma, E.; Sheng, H.W. Atomic level structure in multicomponent bulk metallic glass. Phys. Rev. Lett. 2009, 102, 245501:1-245501:4.

(C) 2012 by the authors; licensee MDPI, Basel, Switzerland. This article is an open access article distributed under the terms and conditions of the Creative Commons Attribution license (http://creativecommons.org/licenses/by/3.0/). 